\title{
Code and Data.
}

The code to perform the simulations and analyze the data generated in this project, and a summary data set are included as a Git repository at https://github. com/gnardin/CRUST. Refer to the description in the main page of this repository for further instructions and details of the implementation. 